\title{
Continuous quaternion based almost global attitude tracking
}

\author{
Thomas Conord $^{1}$ and Dimitri Peaucelle ${ }^{1}$
}

\begin{abstract}
This paper considers the attitude control problem of a generic rotating 3 degrees of freedom fully actuated rigid object. The specific studied problem is the deviation control of this object around a theoretically feasible attitude trajectory. The rotation motion has an intrinsic non linear behaviour (trigonometric, $2 \pi$-periodicity) that need to build non linear and hybrid controllers to get global stability of the closed loop system. This paper considers the opportunity to use the quaternion framework to build a continuous non linear state feedback that reaches an almost global asymptotical stability. Some perspectives to enhance this result with integrators to cancel out static and drag errors are eventually proposed.
\end{abstract}

\section{INTRODUCTION}

This paper studies the attitude control problem of a rigid fully actuated object in the unit quaternion framework. The attitude control of a rigid object is a widely studied problem as it is a central issue of all moving robots: aircrafts, drones, spacecrafts, satellites, manipulators.

The attitude control presents some specific complexity linked to the topology of the rotational motion : its trigonometric behaviour and its $2 \pi$-periodicity. The paper [1] describes quite exhaustively the various mathematical frameworks existing to model the attitude of an object: Euler angles, rotation matrix, unit quaternion. As demonstrated in [2], this topological issue leads to not be able to build a global stable static or dynamic continuous linear time invariant control law for the attitude control of a rigid object.

The unit quaternion framework is chosen here because of its efficiency and compactness: 4 parameters against 9 for the rotation matrix, it does not involve direct trigonometry developments, and it suits the state space framework. The topological issue remains and appears as a singularity problem called double coverage: a unit quaternion $q$ and its opposite $-q$ represents the same object attitude (cf. [3] for detailed quaternion algebra). This quaternion singularity problem may generate an unwinding phenomenon: the controlled object flips back all the way around whereas it was just nearby the required attitude, but it had done the travel rotating from the other side and it does again the same travel backwards.

From the general mathematical "stability of motion" problem which can be formalized with the Lyapunov theory as in [4], the motion control of a rigid object is included in the class of Lagrangian mechanical non linear systems as shown in [5]. For this class of systems,

${ }^{1}$ LAAS-CNRS, University of Toulouse, CNRS, UPS, Toulouse, France. many control strategies have been studied, starting with a reference result [6] in 1988 with a dynamics reinjection input and a Proportional Derivative (PD) deviation controller architecture.

The quaternion-based attitude control problem is still nowadays widely studied. Some approaches study similar strategies as previous Lagrangian PD control architecture [7], [8]. Others build some non linear controllers that match the non linearities and singularities of the quaternion attitude kinematics with specific Lyapunov function [9], [10], [11], [12], [13]. The singularity issue may also be managed with a hybrid control strategy depending on the sign of the first component of the unit quaternion as in [14]. Some others address the robustness of the controller against disturbance input or parametric uncertainties [15], [16], [17].

As developped in section II, the first novelty of this paper is to represent the attitude trajectory deviation dynamics as a single quaternion-based non linear state space model. It brings out a model simplification in section III-A which is another result, which leads to build in section III-B a continuous non linear state feedback that reaches almost global asymptotic stability as defined in [18], framed as a Proportional Derivative controller. The demonstration is performed with a relaxation of conditions of Input to State Stability (ISS) as demonstrated in [19]. We eventually open some perspectives in section III-C to enhance this result with integrators to cancel out static and drag errors.

\section{Attitude DEViation MOdeL}

\section{A. Newton's law for the rotation motion}

The Newton's law for the attitude dynamics applied to the center of gravity of an object of inertia $J_{b} \in \mathbb{R}^{3 \times 3}$, constant symmetric definite positive matrix, corresponds to the following set of differential equations (cf. [3] for details on rotation motion and quaternion):

$$
H:\left\{\begin{array}{l}
\frac{d(q)}{d t}=\frac{1}{2}\left(\begin{array}{c}
-q_{V} \\
S_{q}(q)
\end{array}\right) \omega_{b} \\
J_{b} \frac{d\left(\omega_{b}\right)}{d t}+S_{V}\left(\omega_{b}\right) J_{b} \omega_{b}=C_{a c t_{b}}+C_{e x t_{b}}
\end{array}\right.
$$

With all the variables without index expressed in the earth reference frame, and all the variables with the index " expressed in the rigid body reference frame, with: 
- the instantaneous attitude represented as the unit quaternion:

$$
q=\left(\begin{array}{c}
q_{o} \\
q_{V}
\end{array}\right)=\left(\begin{array}{l}
q_{o} \\
q_{1} \\
q_{2} \\
q_{3}
\end{array}\right)=\left(\begin{array}{c}
\cos (\theta / 2) \\
\sin (\theta / 2)\left(\begin{array}{l}
n_{x} \\
n_{y} \\
n_{z}
\end{array}\right)
\end{array}\right) \in \mathbb{R}^{4}
$$

which corresponds to a rotation of the object by an angle $\theta$ around the axis defined by the unit vector $n=\left(\begin{array}{lll}n_{x} & n_{y} & n_{z}\end{array}\right)^{\top} \in \mathbb{R}^{3}$. $q_{o}$ is generally called the scalar part of the quaternion and $q_{V}$ the vector part. The double coverage issue appears in this definition as $q$ and $-q$ represents the same attitude. A complete non ambiguous attitude position with these terms can be defined by $\operatorname{sign}\left(q_{o}\right) q_{V}$.

- $S_{q}(q)$ is the skew symmetric matrix of the quaternion $q$ defined as follows:

$$
S_{q}(q)=\left(\begin{array}{ccc}
q_{o} & -q_{3} & q_{2} \\
q_{3} & q_{o} & -q_{1} \\
-q_{2} & q_{1} & q_{o}
\end{array}\right)=q_{o} I_{3}+S_{V}\left(q_{V}\right)
$$

The skew symmetric matrix $S_{V}$ of a vector allows to perform the cross-product of two vectors $V_{1}$ and $V_{2}$ of $\mathbb{R}^{3}$ such that : $S_{V}\left(V_{1}\right) V_{2}=V_{1} \wedge V_{2}$.

- $\omega_{b} \in \mathbb{R}^{3}$ is the rotation speed vector,

- $C_{a c t_{b}}, C_{\text {ext }} \in \mathbb{R}^{3}$ are respectively the torques applied by the actuators and by the external environment (air drag, objects or walls in contact with the object).

The second equation of (1) is expressed in the rotating rigid body reference frame so that the inertia appears as the constant $J_{b}$. In this frame, the inputs are also directly equal to actuators actions (actuators attached to the rotating object). We assume to have a fully actuated object, actuators acting independently on the three components of the control input torque $C_{a c t_{b}}$.

\section{B. State space attitude deviation model}

Assumption 1: The optimal command problem (cf. [20]) is considered solved for the theoretical system (1) for a thoretical inertia $J_{b}^{*}$, without any external disturbances $\left(C_{\text {ext }}=0\right)$, giving a theoretically feasible trajectory $\left(q^{*}\right.$, $\left.\omega_{b}^{*}\right)$ with its optimal input $C_{a c t_{b}}^{*}$ solutions of:

$$
H^{*}:\left\{\begin{array}{l}
\frac{d\left(q^{*}\right)}{d t}=\frac{1}{2}\left(\begin{array}{c}
-q_{V}^{*} \\
S_{q}\left(q^{*}\right)
\end{array}\right) \omega_{b}^{*} \\
J_{b}^{*} \frac{d\left(\omega_{b}^{*}\right)}{d t}+S_{V}\left(\omega_{b}^{*}\right) J_{b}^{*} \omega_{b}^{*}=C_{a c t_{b}}^{*}
\end{array}\right.
$$

All the theoretical values are noted with a * exponent.

Proposition 1: The tracking deviation of (1) with respect to (2) has the following almost linear open loop representation:

$$
\hat{\Sigma}:\left\{\begin{aligned}
\dot{x} & =\hat{A}(x) x+B(u+w) \\
z_{q} & =C_{q} x \\
z_{\omega} & =C_{\omega} x \\
y & =x
\end{aligned}\right.
$$

With the state $x=\left(\begin{array}{ll}q_{V}^{\varepsilon \top} & \omega_{b}^{\varepsilon^{*} \top}\end{array}\right)^{\top} \in \mathbb{R}^{6}$ and the state space matrices:

$$
\begin{gathered}
\hat{A}(x)=\left(\begin{array}{cc}
0 & \frac{1}{2} S_{q}\left(q^{\varepsilon}\right) \\
0 & 0
\end{array}\right) \in \mathbb{R}^{6 \times 6}, B=\left(\begin{array}{c}
0 \\
I_{3}
\end{array}\right) \in \mathbb{R}^{6 \times 3} \\
C_{q}=\left(\begin{array}{ll}
I_{3} & 0
\end{array}\right), C_{\omega}=\left(\begin{array}{ll}
0 & I_{3}
\end{array}\right) \in \mathbb{R}^{3 \times 6}
\end{gathered}
$$

The scalar part of the quaternion $q_{o}^{\varepsilon}$ inside the matrix $S_{q}\left(q^{\varepsilon}\right)=q_{o}^{\varepsilon} I_{3}+S_{V}\left(q_{V}^{\varepsilon}\right)$ in $\hat{A}(x)$ is the non linear function of the state solution of:

$$
\frac{d q_{o}^{\varepsilon}}{d t}=-\frac{1}{2} q_{V}^{\varepsilon \top} \omega_{b}^{\varepsilon^{*}}=-\frac{1}{4} x^{\top}\left(\begin{array}{cc}
0 & I_{3} \\
I_{3} & 0
\end{array}\right) x
$$

It also respects the unit norm constraint: $q_{o}^{\varepsilon 2}+\left\|q_{V}^{\varepsilon}\right\|^{2}=1$. $q_{o}^{\varepsilon}$ is not considered as a state of the system.

Proof: The attitude deviation system $\hat{\Sigma}$ is obtained performing the non linear difference between (1) and (2) giving the following variables definitions:

- $x=\left(\begin{array}{ll}q_{V}^{\varepsilon^{\top}} & \omega_{b}^{\varepsilon^{*} \top}\end{array}\right)^{\top} \in \mathbb{R}^{6}$ the state defined by:

- $q_{V}^{\varepsilon}$ the vector part of the attitude error in the quaternion format corresponding to:

$$
q^{\varepsilon}=\left(\begin{array}{c}
q_{o}^{\varepsilon} \\
q_{V}^{\varepsilon}
\end{array}\right)=\left(\begin{array}{cc}
q_{o} & -q_{V} \\
q_{V} & S_{q}(q)
\end{array}\right)\left(\begin{array}{c}
q_{o}^{*} \\
-q_{V}^{*}
\end{array}\right)
$$

Which correponds to $q^{\varepsilon}=q \star q^{*-1}$, with the operation $\star$ corresponding to the quaternion multiplication of the quaternion algebra (cf. [3]). It can also be written : $q=q^{\varepsilon} \star q^{*}$, meaning $q^{\varepsilon}$ is the rotation correction to be performed to bring the object from the theoretical attitude $q^{*}$ to the real current attitude $q$. The object is on the trajectory meaning $q=q^{*}$, if and only if $q^{\varepsilon}=\left(\begin{array}{llll} \pm 1 & 0 & 0 & 0\end{array}\right)^{\top}$, both values (double coverage) representing the same reference attitude corresponding to no rotation correction.

- $\omega_{b}^{\varepsilon^{*}}$ the rotation speed error:

$$
\omega_{b}^{\varepsilon^{*}}=Q_{q}\left(q^{*}\right) \omega_{b}^{\varepsilon}=Q_{q}\left(q^{*}\right)\left(\omega_{b}-\omega_{b}^{*}\right)
$$

The multiplication of the rotation speed error vector $\omega_{b}^{\varepsilon}=\omega_{b}-\omega_{b}^{*}$ by the rotation matrix $Q_{q}\left(q^{*}\right)$ does not change its norm which is equal to: $\left\|\omega_{b}^{\varepsilon}\right\|=$ $\left\|\omega_{b}-\omega_{b}^{*}\right\|=\left\|\omega_{b}^{\varepsilon^{*}}\right\|$. Therefore, when $\omega_{b}^{\varepsilon^{*}}=0$, we get $\omega_{b}=\omega_{b}^{*}$ which corresponds to be on the trajectory for the rotation speed.

The usual rotation matrix $Q_{q}(q) \in \mathbb{R}^{3 \times 3}$ corresponding to the rotation defined by the quaternion $q$, such that any vector $V_{b}$ expressed in the rigid body reference frame is obtained in the earth one as follow: $V_{e}=Q_{q}(q) V_{b}$, can be computed with the following relationship:

$$
Q_{q}(q)=I_{3}+2 q_{o} S_{V}\left(q_{V}\right)+2 S_{V}\left(q_{V}\right)^{2}
$$

- $u$ the correction input defined such that the total torque command $C_{a c t_{b}}$ of (1) is equal to:

$$
\begin{aligned}
& C_{a c t_{b}}=J_{b}^{*} \dot{\omega}_{b}^{*}+S_{V}\left(\omega_{b}^{*}\right) J_{b}^{*} \omega_{b}^{*} \\
& +S_{V}\left(\omega_{b}^{*}\right)\left(2 J_{b}^{*}-\operatorname{Tr}\left(J_{b}^{*}\right) I_{3}\right) \omega_{b}^{\varepsilon}+S_{V}\left(\omega_{b}^{\varepsilon}\right) J_{b}^{*} \omega_{b}^{\varepsilon} \\
& +J_{b}^{*} Q_{q}\left(q^{*}\right)^{-1} u
\end{aligned}
$$


- $w=w_{\Delta}+w_{\text {ext }}$ the perturbation input such that:

- $w_{\Delta}$ is the internal perturbation input generated by the model approximation done when computing the theoretical input command $C_{a c t_{b}}^{*}$ with $J_{b}^{*}=J_{b}-$ $\Delta J_{b}$ :

$$
\begin{aligned}
w_{\Delta}= & Q_{q}\left(q^{*}\right) J_{b}^{-1}\left[-\Delta J_{b} \dot{\omega}_{b}^{*}-S_{V}\left(\omega_{b}^{*}\right) \Delta J_{b} \omega_{b}^{*}\right. \\
& -S_{V}\left(\omega_{b}^{*}\right)\left(2 \Delta J_{b}-T r\left(\Delta J_{b}\right) I_{3}\right) \omega_{b}^{\varepsilon} \\
& \left.-S_{V}\left(\omega_{b}^{\varepsilon}\right) \Delta J_{b} \omega_{b}^{\varepsilon}-\Delta J_{b} Q_{q}\left(q^{*}\right)^{-1} u\right]
\end{aligned}
$$

- $w_{\text {ext }}$ is the external disturbance input due to the external interactions (winds, walls) :

$$
w_{\text {ext }}=Q_{q}\left(q^{*}\right) J_{b}^{-1} C_{e x t_{b}}
$$

- $z_{q}$ the trajectory tracking performance output corresponding to the attitude deviation position $q_{V}^{\varepsilon}$.

- $z_{\omega}$ the trajectory tracking performance output corresponding to the rotation speed deviation $\omega_{b}^{\varepsilon^{*}}$.

- $y=x$ the measurments considered available through physical sensors measures and data fusion post processing such as Kalman filtering (cf. [21]). Thus $q_{o}^{\varepsilon}$ is also available thanks to (4).

Comment: $q_{V}^{\varepsilon}$ describes entirely the attitude position of the object except to a sign ambiguity, the double coverage. To take away this ambiguity, the following complete state may be used for the results analysis:

$$
x_{c}=\left(\operatorname{sign}\left(q_{o}^{\varepsilon}\right) q_{V}^{\varepsilon^{\top}} \quad \omega_{b}^{\varepsilon^{*} \top}\right)^{\top} \in \mathbb{R}^{6}
$$

However, this ambiguity does not affect the definition of the equilibrium point : it is the single value $x^{o}=$

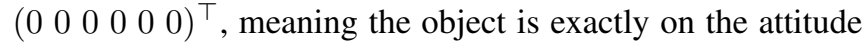
trajectory when the state is zero. With this definition of deviation model $\hat{\Sigma}$, we can thus look for a structure of controller that makes the single point $x^{o}=0$ asymptotically stable.

\section{ATtITUdE TRACKING CONTROL}

\section{A. Preliminaries}

As confirmed with the proof of the below lemma 1, despite the coupling of the three rotation speeds directions due to the term $S_{V}\left(q_{V}^{\varepsilon}\right)$ in the matrix $\hat{A}(x)$, the deviation system (3) appears to be homogenous in the three directions of space and can be studied according to this simplification:

Definition 1: Let the reduced non linear second order system of (3) be:

$$
\hat{\Sigma}_{r}:\left\{\begin{array}{l}
\dot{x}_{r}=\hat{A}_{r}\left(x_{r}\right) x_{r}+B_{r}\left(u_{r}+w_{r}\right) \\
z_{q_{r}}=C_{q_{r}} x_{r} \\
z_{\omega_{r}}=C_{\omega_{r}} x_{r} \\
y_{r}=x_{r}
\end{array}\right.
$$

With $x_{r}=\left(\begin{array}{ll}q_{V_{r}}^{\varepsilon} & \omega_{b_{r}}^{\varepsilon^{*}}\end{array}\right)^{\top}=\left(\sin \left(\theta^{\varepsilon} / 2\right) \quad \dot{\theta}^{\varepsilon}\right)^{\top} \in \mathbb{R}^{2}$, $u_{r}, w_{r} \in \mathbb{R}$ the scalar inputs projections of the original inputs $u$ and $w$ on the instantaneous axis $n^{\varepsilon}\left(n^{\varepsilon}(t) \in \mathbb{R}^{3}\right.$ the vector defined by $\left.q_{V}^{\varepsilon}=\sin \left(\theta^{\varepsilon} / 2\right) n^{\varepsilon}\right)$, and the matrices

$$
\begin{gathered}
\hat{A}_{r}\left(x_{r}\right)=\left(\begin{array}{cc}
0 & 1 / 2 q_{o}^{\varepsilon} \\
0 & 0
\end{array}\right) \in \mathbb{R}^{2 \times 2}, B_{r}=\left(\begin{array}{l}
0 \\
1
\end{array}\right) \in \mathbb{R}^{2 \times 1} \\
C_{q_{r}}=\left(\begin{array}{ll}
1 & 0
\end{array}\right) \in \mathbb{R}^{1 \times 2}, C_{\omega_{r}}=\left(\begin{array}{ll}
0 & 1
\end{array}\right) \in \mathbb{R}^{1 \times 2}
\end{gathered}
$$

Comment: $\hat{\Sigma}_{r}$ corresponds to the projection of $\hat{\Sigma}$ on the direction of the unit vector $n^{\varepsilon}$, which is the 1-dimension control problem, meaning the control of an object rotating around one single fixed axis.

\section{Lemma 1: A time varying state feedback}

$u_{r}=\left[-k_{p}\left(t, x_{r}\right)-k_{d}\left(t, x_{r}\right)\right] x_{r}, k_{p}\left(t, x_{r}\right), k_{d}\left(t, x_{r}\right) \in \mathbb{R}$

makes $x_{r}=0$ asymptotically stable for the reduced system (12) and $V_{r}\left(t, x_{r}\right)=x_{r}^{\top} P_{r}\left(t, x_{r}\right) x_{r}$, with $P_{r}\left(t, x_{r}\right) \in \mathbb{R}^{2 \times 2}$ a time varying symmetric definite positive matrix, is a Lyapunov function that demonstrates it,

if and only if the state feedback

$$
u=\left[-k_{p}(t, x) I_{3}-k_{d}(t, x) I_{3}\right] x
$$

makes $x=0$ asymptotically stable for the original deviation system (3) and the Lyapunov function $V(t, x)=x^{\top} P(t, x) x$ with $P(t, x)=P_{r}(t, x) \otimes I_{3}$ is a certificate.

Proof: Let us note whithout hat, respectively $A_{r}$ and $A$, the closed loop state matrices with this state feedback. Let us notice that $A$ can be decomposed as follow :

$$
A(x)=A_{r}(x) \otimes I_{3}+A_{V}(x)
$$

with $\otimes$ the Kronecker product and with

$$
A_{V}=\left(\begin{array}{cc}
0 & 1 / 2 S_{V}\left(q_{V}^{\varepsilon}\right) \\
0 & 0
\end{array}\right)
$$

which verifies for any symmetric matrix $P(t, x)=P_{r}(t, x) \otimes$ $I_{3}$

$$
x^{\top}\left(A_{V}(x)^{\top} P(t, x)+P(t, x) A_{V}(x)\right) x=0
$$

as $S_{V}\left(q_{V}^{\varepsilon}\right)$ corresponds to a vectorial product of $q_{V}^{\varepsilon}$ with $\omega_{b}^{\varepsilon^{*}}$ which result is orthogonal to $q_{V}^{\varepsilon}$ and $\omega_{b}^{\varepsilon^{*}}$; and as $S_{V}\left(q_{V}^{\varepsilon}\right)^{\top}=-S_{V}\left(q_{V}^{\varepsilon}\right)$ (skew symmetric matrix).

This leads to:

$$
\dot{V}=x^{\top}\left(A(x)^{\top} P(t, x)+P(t, x) A(x)+\dot{P}(t, x)\right) x
$$

$\dot{V}=x^{\top}\left(\left(A_{r}(x)^{\top} P_{r}(t, x)+P_{r}(t, x) A_{r}(x)+\dot{P}_{r}(t, x)\right) \otimes I_{3}\right) x$

Therefore, $\dot{V}<0$ if and only if $\dot{V}_{r}<0$, the lemma is proved.

Comments: This controller structure is similar to a Proportional Derivative controller with respect to the attitude position $q_{V}^{\varepsilon}$ and the rotation speed $\omega_{b}^{\varepsilon^{*}}$, that is why the controller parameters are noted $k_{p}$ as a "proportional" gain, and $k_{d}$ as a "derivative" gain. When $k_{p}$ and $k_{d}$ are taken constant, this controller is close to common quaternion based control approaches developed in other papers, as in 
[22].

This result brings out the fact that we can look for scalar structure of controller for the original deviation system (3) and study its stability through the reduced system (12).

\section{B. Almost global asymptotical stable state feedback}

Definition 2: As defined in [18], an autonomous system defined by $\dot{x}=f(x)$, where $f: \mathbb{R}^{n} \mapsto \mathbb{R}^{n}$ is $\mathcal{C}^{1}$ (enough to ensure the existence and uniqueness of solutions to the initial value problem) and $f(0)=0$, is almost global asymptotical stable if all the trajectories but a reduced set of zero Lebesgue measure converge asymptotically to the origin.

Using previous lemma 1 and inspired from the hybrid control developped in [14] reaching a global stability property, we consider to manage the double coverage sign ambiguity of $q_{V}^{\varepsilon}$ with a continuous multiplication by $q_{o}^{\varepsilon}$, which is of the needed sign to inverse the command when the object crosses backside.

Proposition 2: A non linear state feedback controller of the form:

$$
u(x)=-2 k_{p} q_{o}^{\varepsilon} q_{V}^{\varepsilon}-k_{d} \omega_{b}^{\varepsilon^{*}}=K(x) x
$$

giving, for the original deviation system (3), the closed loop non linear system without perturbations $(w=0)$ :

$$
\begin{aligned}
\Sigma: \dot{x} & =A(x) x=(\hat{A}(x)+B K(x)) x \\
& =\left(\begin{array}{cc}
0 & 1 / 2 S_{q}\left(q^{\varepsilon}\right) \\
-2 k_{p} q_{o}^{\varepsilon} I_{3} & -k_{d} I_{3}
\end{array}\right) x
\end{aligned}
$$

with $K(x)=\left(-2 k_{p} q_{o}^{\varepsilon} I_{3}-k_{d} I_{3}\right), k_{p}, k_{d}>0$ scalar constants, makes this closed loop system almost global asymptotical stable with a compact invariant set defined by

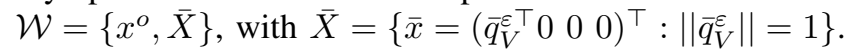

Comments: the exact flipped backside attitudes set $\bar{X}$ (corresponding to $q_{o}^{\varepsilon}=0$ ) from $x^{o}=0$, rotating around any axis, is a set of unstable equilibrium points with the controller (14). Indeed, the proportional term decreases up to vanish its authority to bring back the object to $x^{o}=0$ when the object is around the unstable equilibrium points. It allows not to create conditions for chattering around the unstable equilibrium points. Moreover, these points are at the opposite from a tracking tolerance tube in which a controller shall maintain the object (meaning far away from the actuators range sizing). Thus this controller structure could also be a good strategy to deal smoothly with actuators saturation.

The multiplication by 2 of the proportional like term gives the 1-dimension closed loop system nice formulation (directly equivalent to the reduced system (12) in closed loop):

$$
\ddot{\theta}^{\varepsilon}=-k_{p} \sin \left(\theta^{\varepsilon}\right)-k_{d} \dot{\theta}^{\varepsilon}+w_{r}
$$

Proof: The proof of the propostion 2 is performed using the properties of almost global asymtotic stability as defined in [18], previously mentioned as a dual to Lyapunov's stability theorem [23], which is a derivative of the Lyapunov stability theorem [4] for systems with several equilibria or invariant sets. We precisely use directly the recent derivative result presented in [19] which is a relaxation of conditions of ISS for multi stable periodic systems (the example in [19] is precisely the demonstration of the stability of the 1-dimension control problem).

One may define for any $\alpha \in] 0 ; 1[$, the class of Lyapunov function $V(x)=x^{\top} P(x) x$ with the matrix $P(x)$ affine in $q_{o}^{\varepsilon}$ equal to:

$$
P(x)=\left(\begin{array}{cc}
4\left(k_{p}+\alpha k_{d}^{2}\right) I_{3} & 2 \alpha k_{d} q_{o}^{\varepsilon} I_{3} \\
2 \alpha k_{d} q_{o}^{\varepsilon} I_{3} & I_{3}
\end{array}\right) \succ 0
$$

The derivative of the Lyapunov function $V(x)$ for the trajectories of the closed loop system (15) is equal to:

$$
\begin{aligned}
& \dot{V}(x)=x^{\top}\left[A(x)^{\top} P(x)+P(x) A(x)+\dot{P}(x)\right] x \\
& =x^{\top}\left(\begin{array}{cc}
-8 \alpha k_{p} k_{d} q_{o}^{\varepsilon 2} I_{3} & 0 \\
0 & -2 k_{d}\left(1-\alpha q_{o}^{\varepsilon}\right) I_{3}
\end{array}\right) x \\
& -8 \alpha k_{d}\left(\dot{q}_{o}^{\varepsilon}\right)^{2}<0 \quad \forall x \notin \mathcal{W}
\end{aligned}
$$

From the expression of $V$ and $\dot{V}$ and the definition 7 of [19], we can see that $V$ is an ISS Lyapunov function. Therefore it is a practical ISS Leonov function (def. 11 of [19]), which is equivalent to have the closed loop system (15) ISS with respect to the invariant set $\mathcal{W}$ (cf. theorem 14 of [19]), thus the almost global asymptotic stability property.

The figure 1 gives an equivalent physical assembly composed of a rotating cylinder in friction inside another cylinder, with a spring attached to both cylinders bringing back the rotating cylinder to a reference position, representing the action of the "Proportional Derivative" like controller for the 1-dimension rotation control problem.

A representation in the phase plane of the reduced system (12) in closed loop with this controller for the complete state $x_{c}$ defined by (11) $\left(q_{V}^{\varepsilon}\right.$ corrected with the sign of $\left.q_{o}^{\varepsilon}\right)$ is given figure 2 .

\section{Perspectives of almost global stability with integrators}

This result of state feedback (14) highlights the special interest of the quantity $q_{o}^{\varepsilon}$ to build controllers for the attitude deviation system (3). In some way, $\left|q_{o}^{\varepsilon}\right|$ looks like a Lyapunov dual function (cf. a dual to a Lyapunov stability theorem [23]): it is equal to 1 when $x=0$, and 0 when the object is at the opposite.

Thus the multiplication by $q_{o}^{\varepsilon}$ has no effect when the object is at the equilibrium point; it enables to inhibit some part of the command when the object is flipped backside; and its sign allows to switch the direction of the terms of 


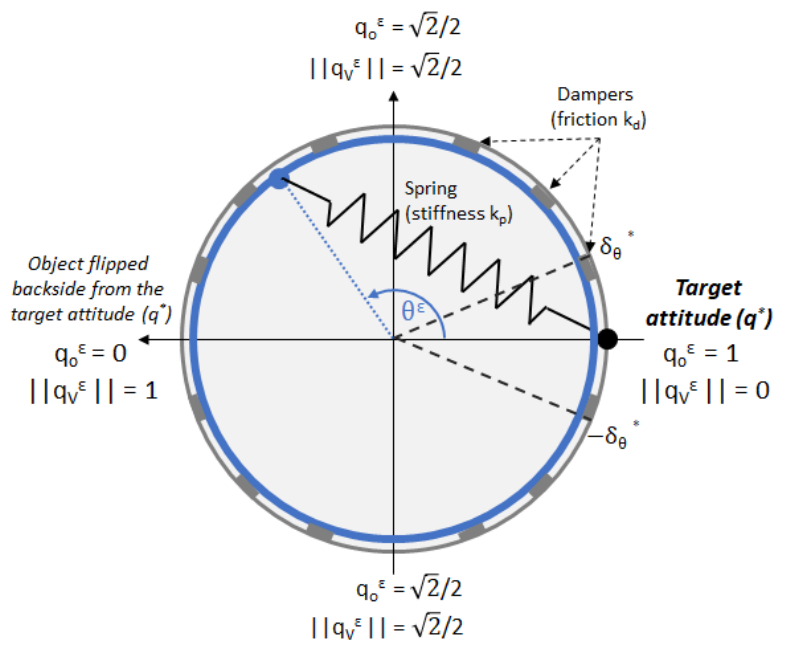

Fig. 1. Equivalent physical assembly representing the behaviour of the reduced system in closed loop with the proportional derivative like attitude controller (14).

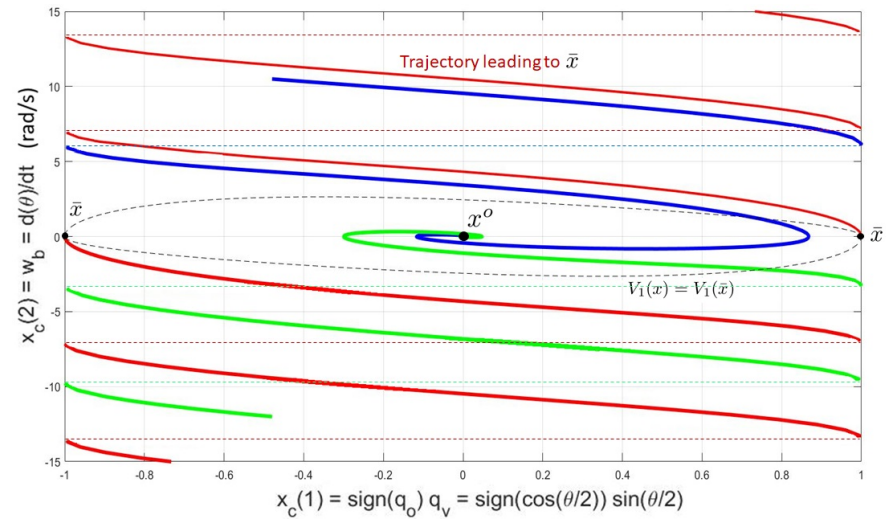

Fig. 2. Phase plane of the reduced system in closed loop with the proportional derivative like attitude controller (14), with $k_{p}=1$ and $k_{d}=1$.

the command that need to switch when the object crosses backside.

As the system (3) undergoes input perturbations $w_{\Delta}$ due to model approximations when computing the optimal command, there will be a static error with such a Proportional Derivative controller structure (14). Besides, this perturbation is variable and added to the variable external perturbations $w_{\text {ext }}$ mainly due to the aerodynamics drag. For instance, these variations can be strictly increasing for some time intervals where the object is performing some specific maneuvers, generating some drag errors. Depending on the number of levels of errors (static $+N-1$ levels of drag errors) required to be cancelled out, we may then add $N$ integrators to the controller.

The integrators, which main function in our case is to delete errors, are useful near the trajectory. Therefore, they could be "switched off" when the object is at the opposite of the equilibrium point: using previous property of $q_{o}^{\varepsilon}$, it could be done for example with a multiplication by an even power of $q_{o}^{\varepsilon}\left(q_{o}^{\varepsilon 2}, q_{o}^{\varepsilon 4}, \ldots\right)$.

Conjecture 1: A linear Proportional N-Integral Derivative (P N-I D) controller, of the form:

$u\left(x_{N}\right)=-k_{i_{N}} q_{o}^{\varepsilon 2 N} \eta_{N}-\ldots-k_{i_{1}} q_{o}^{\varepsilon 2} \eta_{1}-2 k_{p} q_{o}^{\varepsilon} q_{V}^{\varepsilon}-k_{d} \omega_{b}^{\varepsilon^{*}}$

with $\dot{\eta}_{1}=2 q_{o}^{\varepsilon} q_{V}^{\varepsilon}$ and $\dot{\eta}_{k+1}=q_{o}^{\varepsilon 2 k} \eta_{k}, \forall k=1 . . N-1$, giving, for the original deviation system (3), the closed loop non linear system without perturbations $(w=0)$ :

$\Sigma_{N}: \dot{x}_{N}=A_{N}\left(x_{N}\right) x_{N}=\hat{A}_{N}\left(x_{N}\right) x_{N}+B_{N} u\left(x_{N}\right)$

with the state $x_{N}=\left(\eta_{N}^{\top} \ldots \eta_{1}^{\top} q_{V}^{\varepsilon^{\top}} \omega_{b}^{\varepsilon^{*} \top}\right)^{\top}$ and the closed loop matrix

$$
\begin{aligned}
A_{N}\left(x_{N}\right)= & \left(R_{q_{o N}}\left(q_{o}^{\varepsilon}\right) R_{2_{N}}^{-1} A_{N}^{o} R_{2_{N}} R_{q_{o N}}\left(q_{o}^{\varepsilon}\right)\right) \otimes I_{3} \\
& +A_{V_{N}}\left(x_{N}\right)
\end{aligned}
$$

with

$$
\begin{aligned}
& A_{N}^{o}=\left(\begin{array}{cccccc}
0 & 1 & \ldots & 0 & 0 & 0 \\
\ldots & \ldots & \ldots & \ldots & \ldots & \ldots \\
0 & 0 & \ldots & 0 & 1 & 0 \\
0 & 0 & \ldots & 0 & 0 & 1 \\
-k_{i_{N}} & -k_{i_{N-1}} & \ldots & -k_{i_{1}} & -k_{p} & -k_{d}
\end{array}\right) \\
& R_{2_{N}}=\left(\begin{array}{cccccc}
1 & 0 & \ldots & 0 & 0 & 0 \\
0 & 1 & \ldots & 0 & 0 & 0 \\
\cdots & \cdots & \cdots & \cdots & \cdots & \cdots \\
0 & 0 & \cdots & 1 & 0 & 0 \\
0 & 0 & \cdots & 0 & 2 & 0 \\
0 & 0 & \ldots & 0 & 0 & 1
\end{array}\right) \\
& R_{q_{o N_{L}}}\left(q_{o}^{\varepsilon}\right)=\left(\begin{array}{cccccc}
1 & 0 & \ldots & 0 & 0 & 0 \\
0 & 1 & \ldots & 0 & 0 & 0 \\
\ldots & \ldots & \ldots & \ldots & \ldots & \ldots \\
0 & 0 & \ldots & 1 & 0 & 0 \\
0 & 0 & \ldots & 0 & q_{o}^{\varepsilon} & 0 \\
0 & 0 & \ldots & 0 & 0 & 1
\end{array}\right) \\
& R_{q_{o N}}\left(q_{o}^{\varepsilon}\right)=\left(\begin{array}{cccccc}
q_{o}^{\varepsilon 2 N} & 0 & 0 & \ldots & 0 & 0 \\
0 & q_{o}^{\varepsilon 2(N-1)} & 0 & \ldots & 0 & 0 \\
\ldots & \ldots & \ldots & \ldots & \ldots & \ldots \\
0 & 0 & 0 & \ldots & q_{o}^{\varepsilon} & 0 \\
0 & 0 & 0 & \ldots & 0 & 1
\end{array}\right) \\
& A_{V_{N}}\left(x_{N}\right)=\left(\begin{array}{ccccc}
0 & \ldots & 0 & 0 & 0 \\
0 & \ldots & 0 & 0 & 0 \\
\ldots & \ldots & \ldots & \ldots & \ldots \\
0 & \ldots & 0 & 0 & 0 \\
0 & \ldots & 0 & 0 & 1 / 2 S_{V}\left(q_{V}^{\varepsilon}\right) \\
0 & \ldots & 0 & 0 & 0
\end{array}\right)
\end{aligned}
$$

with $A_{N}^{o}$ a constant Hurwitz stable matrix, may make $x_{N}^{o}=0$ almost global asymptotical stable for the closed loop system $\Sigma_{N}$, without static errors nor levels 1 to $N-1$ drag errors on the output $z_{q}=q_{V}^{\varepsilon}$.

Partial proof: The demonstration of integrators canceling out levels of errors for LTI system extended to this non linar system is not presented in detail : it can be noticed that this non linear system is framed as an LTI one and that in steady state, $q_{o}^{\varepsilon}$ is equal to 1 and makes the state matrix an LTI one. 
Besides, the proof of stability is performed only for the local stability. No Lyapunov function was find to demonstrate the almost global stability of $x_{N}^{o}=0$ for $\Sigma_{N}$.

Using the decomposition of the closed loop state space matrix $A_{N}\left(x_{N}\right)$, as $A_{N}^{o}$ is assumed Hurwitz stable, it exists a symmetric matrix $P_{N} \succ 0$ such that:

$$
P_{N} A_{N}^{o}+A_{N}^{o \top} P_{N}=Q_{N}^{o} \prec 0
$$

Using the certificate $R_{2_{N}} P_{N} R_{2_{N}}$ with the Lyapunov function $V_{N}\left(x_{N}\right)=x_{N}^{\top} R_{2_{N}} P_{N} R_{2_{N}} x_{N}^{\top}$, the derivative of this Lyapunov function along the trajectory of the system (20) gives the following matrix relationships (as explained in the proof of lemma $1, A_{V_{N}}\left(x_{N}\right)$ disappear in the derivative of the Lyapunov function):

$$
\begin{aligned}
& \quad R_{2_{N}} P_{N} R_{2_{N}} A_{N}\left(x_{N}\right)+A_{N}\left(x_{N}\right)^{\top} R_{2_{N}} P_{N} R_{2_{N}} \\
& =R_{2_{N}}\left(P_{N} R_{q_{o N}} A_{N}^{o} R_{q_{o N}}+R_{q_{o} N_{R}} A_{N}^{o \top} R_{q_{o N}} P_{N}\right) R_{2_{N}} \\
& =R_{2_{N}} Q\left(q_{o}^{\varepsilon}\right) R_{2_{N}} \\
& \text { with } Q\left(q_{o}^{\varepsilon}\right) \text { a continuous function of } q_{o}^{\varepsilon} \text { which is equal } \\
& \text { to } Q\left(q_{o}^{\varepsilon}=1\right)=Q_{N}^{o} \prec 0 \text { at the equilibrium point, as } \\
& R_{q_{o N}}\left(q_{o}^{\varepsilon}=1\right)=I \text { and } R_{q_{o N}}\left(q_{o}^{\varepsilon}=1\right)=I .
\end{aligned}
$$

Therefore, it exists a neighbourhood of $x_{N}=0$, which is equivalent to $q_{o}^{\varepsilon}=1$, such that $\dot{V}_{N}\left(x_{N}\right)<0$. The local stability is proved.

Comments: even though the almost global stability is not proved, the overall structure which is locally stable, with integrators which are smoothly inhibited and reversed when the object goes backside, makes this controller a good candidate. Some simulations with arbitrary Hurwitz stable $A_{N}^{o}$ give similar results of converging trajectories as presented in the phase plane figure 2, whithout being able to figure out trajectories that would be unstable or lead to the unstable equilibrium points; that's why no additional simulation are presented here.

\section{CONCLUSION}

This paper presents a non linear state feedback which allows to perform almost global asymptotical stable tracking of a given feasible attitude trajectory for the attitude deviation control problem. The state feedback structure follows from a model simplification result that enables to frame it as a proportional derivative like controller. Hence, this controller leaves room to static and drag errors necessarily present for the real system with uncertainties and perturbations. Thus we eventually propose in a conjecture some perspectives to enhance this controller structure with integrators. The almost global stability is not demonstrated and deserves to be more investigated in further work. Though, the proved local stability already allows to perform the tracking in a given tolerance tube around the trajectory without errors. Some further work to tune these controllers according to a multi-performance specification is already started in [24], extending to non linear systems some results of the LMI robust control S-Variable approach of [25] dedicated to uncertain LTI systems.

\section{REFERENCES}

[1] N. Chaturvedi, A. Sanyal, and N. McClamroch, "Rigid-body attitude control," IEEE Control Systems, 2011.

[2] S. Bhat and D. Bernstein, "A topological obstruction to continuous global stabilization of rotational motion and the unwinding phenomenon," Systems and Control Letters, 2000.

[3] F. Monteghetti, "Quaternions, orientation et mouvement," Rapport de recherche ISAE-SUPAERO hal-01618257, 2012.

[4] W. Hahn, Stability of Motion, S. V. Berlin, Ed., 1967.

[5] R. M. Murray, "Nonlinear control of mechanical systems: A lagrangian perspective," International Federation of Automatic Control, vol. 21, pp. 31-42, 1997.

[6] B. Paden and R. Panja, "Globally asymptotically stable 'pd+' controller for robot manipulators," International Journal of Control, vol. 47 Issue 6, 1988.

[7] J. Liu and J. Yang, "Spacecraft high accuracy attitude control by quaternion-based nonlinear dynamic inversion," 38th Chinese Control Conference, 2019.

[8] K. A. Ackerman, I. M. Gregory, and N. Hovakimyan, "Flight control methods for multirotor uas," International Conference on Unmanned Aircraft Systems (ICUAS), 2019.

[9] D. Xu, J. He, A. Sheng, Z. Chen, and D. Wang, "Robust attitude tracking control of a rigid spacecraft based on nonlinearly controlled quaternions," 11th Asian Control Conference, 2017.

[10] C. He and J. Huang, "Trajectory tracking and disturbance rejection for quadrotor helicopter by a novel method," 38th Chinese Control Conference, 2019.

[11] T. Ye and C. Yuanli, "Quaternion based sliding mode attitude design for spacecraft," IEEE 3rd ICCSSE, 2017.

[12] Z. Yu and G. Li, "Quaternion-based robust sliding mode control for spacecraft attitude tracking," IEEE 31st Chinese Control and Decision Conference, 2019.

[13] H. Abaunza and P. C. Garcia, "Quadrotor aggressive deployment, using a quaternion-based spherical chattering-free sliding-mode controller," IEEE Transactions on Aerospace and Electronic Systems, vol. 56, no. 3, pp. 1979-1991, 2020.

[14] C. G. Mayhew, R. G. Sanfelice, and A. R. Teel, "Quaternion-based hybrid control for robust global attitude tracking," IEEE Transactions on Automatic Control, vol. 56 No. 11, 2011.

[15] H. Liu, X. Wang, and Y. Zhong, "Quaternion-based robust attitude control for uncertain robotic quadrotors," IEEE TRANSACTIONS ON INDUSTRIAL INFORMATICS, vol. 11 No. 2, 2015.

[16] L. Fan, H. Huang, L. Sun, and K. Zhou, "Robust attitude control for a rigid-flexible-rigid microsatellite with multiple uncertainties and input saturations," Aerospace Science and Technology, vol. 95 105443, 2019.

[17] D. Mihailescu-Stoica, R. Acuna, and J. Adamy, "High performance adaptative attitude control of a quadrotor," 18th European Control Conference, 2019.

[18] P. Monzon and R. Potrie, "Local and global aspects of almost global stability," 2006.

[19] D. Efimov, J. Schiffer, N. Barabanov, and R. Ortega, "Relaxing the conditions of ISS for multistable periodic systems," IFACPapersOnLine, vol. 50, no. 1, pp. 7217 - 7222, 2017, 20th IFAC World Congress.

[20] V. Alekseev, V. Alexéev, V. Tikhomirov, and Fomine, Commande optimale, M. . Mir, Ed., 1982.

[21] F. L. Markley, "Attitude error representations for kalman filtering," Journal of guidance, control, and dynamics, vol. 26, no. 2, p. 311-317, 2003.

[22] R. Shlanbusch, A. Loria, and P. J. Nicklasson, "On the stability and stabilization of quaternion equilibria of rigid bodies," Automatica, vol. 48 (2012) 3135-3141, 2012.

[23] A. Rantzer, "A dual to Lyapunov's stability theorem," Systems and Control Letters, vol. 42, no. 3, pp. 161-168, 2001.

[24] T. Conord and D. Peaucelle, "Multi-performance state-feedback for time-varying linear systems," IFAC MICNON 2021, 2021.

[25] Y. Ebihara, D. Peaucelle, and D. Arzelier, S-Variable Approach to LMI-Based Robust Control, Springer, Ed., 2015. 